\title{
Why are some people's names easier to learn than others? The effects of face similarity on memory for face-name associations
}

\author{
Peter C. Pantelis and Marieke K. van Vugt \\ University of Pennsylvania, Philadelphia, Pennsylvania \\ Robert SEKULER \\ Brandeis University, Waltham, Massachusetts \\ Hugh R. WiLSON \\ York University, Toronto, Ontario, Canada \\ AND \\ Michael J. Kahana \\ University of Pennsylvania, Philadelphia, Pennsylvania
}

\begin{abstract}
Using synthetic faces that varied along four perceptual dimensions (Wilson, Loffler, \& Wilkinson, 2002), we examined the effects of face similarity on memory for face-name associations. The nature of these stimuli allowed us to go beyond the categorical similarity manipulations used in previous verbal associative memory studies to trace out the parametric relation between similarity and various performance measures. In Experiment 1, we found that recall performance diminished as a function of how many studied faces were in the vicinity of the cue face in similarity space. Also, incorrect recalls were more likely to come from nearby positions in face space. Experiments 2 and 3, respectively, demonstrated analogous effects with a set of more distinguishable, photorealistic faces, and in an associative recognition task. These results highlight the similarity between associative recall and associative recognition, and between face-name association and other domains of associative memory.
\end{abstract}

In the study of associative memory, similarity has served as one of the major factors that influences recall performance. High levels of within-pair similarity facilitate recall, whereas high levels of between-pair similarity impair recall (Keppel, 1968; McGeoch, 1942; Osgood, 1949; Robinson, 1927; Skaggs, 1925). The negative effects of between-pair similarity on recall are often understood in terms of the construct of interference. Specifically, if items are represented as vectors of attributes, then the degree to which nontarget representations are activated should be determined by their degree of overlap with the attributes of either the cue or the target (Gibson, 1940; Murdock, 1989).

In the verbal memory literature, studies of associative interference have typically manipulated similarity as a categorical variable, with similar items belonging to the same category or sharing a single critical feature (Feldman \& Underwood, 1957; Levitt \& Goss, 1961; Newman \& Buckhout, 1962; Underwood, 1953). Although it would be ideal to parametrically assess similarity effects in associative memory, verbal materials do not readily lend themselves to such manipulations. Unlike with verbal stimuli, the similarity relations among perceptually defined stimuli, such as faces, can be parametrically varied (Wilson, Loffler, \& Wilkinson, 2002). In addition, the relatively high-dimensional nature of faces as stimuli makes them more easily distinguishable and nameable than other classes of perceptual stimuli (e.g., Kahana \& Sekuler, 2002), and the task of putting a name to a face is indeed both natural and commonplace. Our primary goal in the present study was to systematically examine the role of face similarity in both cued recall and associative recognition of face-name pairs. In studying both cued recall and associative recognition, we also sought to test the hypothesis that similarity effects obey common principles in these two domains of associative memory (Hockley, 2008; Nobel \& Shiffrin, 2001; Verde \& Rotello, 2004; Yonelinas, 1997).

In a study of associative interference for face-name pairs, Fraas et al. (2002) examined the errors that participants made as they attempted to recall names when cued with faces. Overall, extralist intrusions tended to be phonologically similar to the target name (meaning that the intrusion and target names tended to share two or more

\section{J. Kahana, kahana@psych.upenn.edu}


consonants or a vowel-consonant sequence), whereas intralist intrusions (ILIs) tended to be phonologically dissimilar. This study showed the effect of name similarity on memory for face-name associations. Although face similarity has been widely shown to influence performance in item recognition tasks (Busey \& Tunnicliff, 1999; Knapp, Nosofsky, \& Busey, 2006; Yotsumoto, Kahana, Wilson, \& Sekuler, 2007), to our knowledge this factor has not been studied in the domain of face-name associations.

In verbal studies, similarity effects have been studied in terms of both interpair and intrapair similarity. In a study that explored interpair similarity, Bower, ThompsonSchill, and Tulving (1994) had participants learn A-B, then $\mathrm{A}-\mathrm{C}$ word pairs in a modified free recall (MMFR) paradigm. A and B were always from the same category (e.g., apple-banana), and the authors examined how the number of other words in the study list of the same semantic category as A and B affected participants' ability to correctly recall B (banana) when cued with A (apple). The more same-category words in the study list, the worse the initial learning and subsequent retention of the A-B association. Additionally, Nelson, Bajo, McEvoy, and Schreiber (1989) found that when a cue word had more and stronger semantic associates, the probability of sampling the target item at test was reduced (see also Nelson, McKinney, Gee, \& Janczura, 1998, for a related study of verbal association).

Building on these studies of interpair similarity, we asked whether an analogous effect of neighborhood density would occur with memory for face-name pairs. However, we went beyond these previous studies in several ways. First, we explored the role of perceptual similar- ity among visual, nonverbal stimuli (faces), rather than similarity between verbal stimuli based on phonetic or semantic relationships. Also, whereas the Bower et al. (1994) study assessed similarity on the basis of how many same-category competitors a given cue had, ours explores within-category similarity, conceiving of the perceived similarity between any two faces as existing along a continuous metric. Finally, in both our recall and recognition experiments we examined the effect of interstimulus similarity on both accuracy and latency measures.

We explored neighborhood density effects in both cued recall and associative recognition, tasks for which associative interference effects are not necessarily predicted or observed to be the same (Postman, 1976). We hypothesized that a face's name will be more difficult to learn if that face is studied among many perceptually similar faces.

\section{EXPERIMENT 1}

We first examined learning of face-name associations in a cued recall task. The principal motivation of this experiment was to determine whether participants' recall performance would vary with the number of other faces in the study set similar to the cue face.

\section{Method}

Participants. Participants were 25 undergraduate and graduate students (11 male and 14 female) who participated for payment. Each participated in one hour-long session.

Stimuli. We used a set of achromatic male faces (for examples, see Figure 1A). The face stimuli were designed to vary along the 4 principal components of a 37-dimensional face space (Wilson et al., 2002). This face space had been created by taking 37 mea-
A

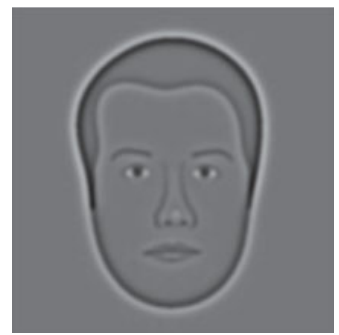

TOM

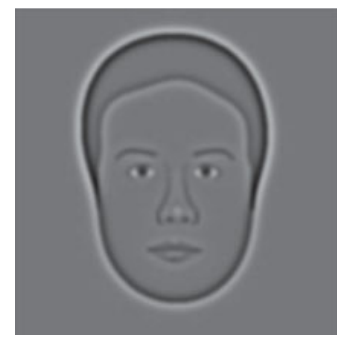

ROB

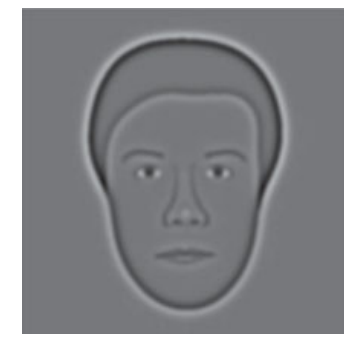

MARK

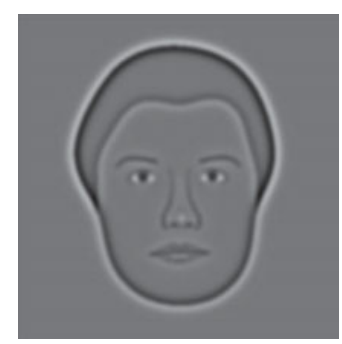

JIM

B

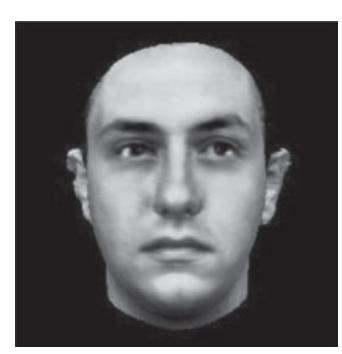

DAN

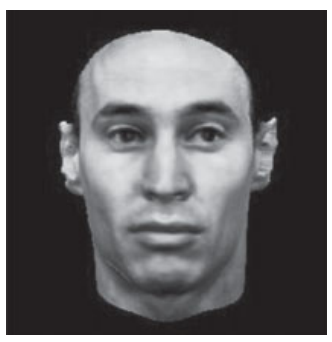

$\mathrm{JOHN}$

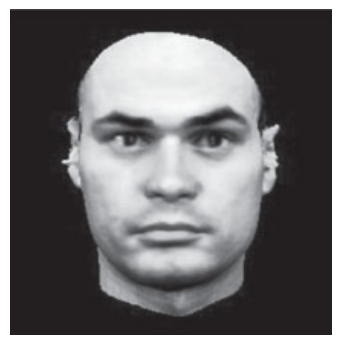

PAUL

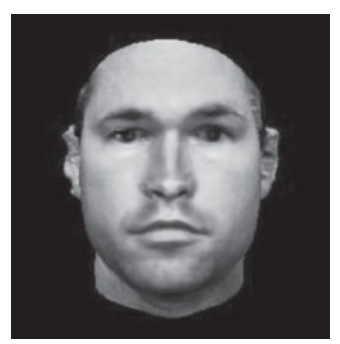

$\mathrm{RICH}$

Figure 1. Sample face-name pairs from (A) Experiments 1 and 3 and (B) Experiment 2. 
surements on a set of photographs of Caucasian males (normalized to have the same head radius), then reconstructing the faces from the principal components extracted from the vector of these measurements. The resulting faces were low-pass filtered at 10 cycles per face width, which is optimal for face processing and which removes components like hair color, hair curliness, and skin texture (Wilson et al., 2002). The advantages of using this face set are that, although the faces are well controlled, they still can be identified with high accuracy (Wilson et al., 2002), and it is possible to measure their interitem similarity precisely. Moreover, the faces are realistic enough to generate responses in the fusiform face area (Loffler, Yourganov, Wilkinson, \& Wilson, 2005), a face-sensitive area in the brain.

The present experiment used only a 4-dimensional (4-D) subset of this 37-dimensional face space. Our stimulus set of 16 faces was created from all permutations of steps of 1 standard deviation (SD) away from the mean face (of the 37-dimensional face space) in the directions of each of the four principal components (1 SD is the threshold for $75 \%$ correct discrimination between two faces that are flashed for $110 \mathrm{msec}$ ).

In order to determine how our particular face set's dimensions were perceived, a separate multidimensional scaling (MDS) study was performed (also reported in van Vugt, Sekuler, Wilson, \& Kahana, 2008). In this MDS study, 23 participants served for two sessions, in which they saw all combinations of three faces (triads) twice, and had to determine the "odd one out." From these ratings, a similarity matrix was constructed by increasing the similarity value for each of the two faces not chosen (see Kahana \& Bennett, 1994, for details). This similarity matrix was transformed into similarity coordinates for every face, using individual differences multidimensional scaling (INDSCAL/ALSCAL; Takane, Young, \& de Leeuw, 1977).

The experimenters derived 4-D stimulus coordinates for each of the 16 faces. Four dimensions was a good fit according to an inspection of the scree plot. This 4-D fit also corresponded to the number of dimensions on the basis of which the faces had been generated.
Figure 2 shows the coordinates of the faces in the MDS-derived space. Because the faces were generated from a set of physical measurements, we can use these measurements to help interpret the meaning of the four dimensions obtained. The first MDS dimension correlated significantly with nose width $[r(15)=.89, p<.001]$ and one of the components of the head shape $[r(15)=.88, p<.01]$. The second MDS dimension correlated significantly with parameters representing the geometry of the hairline $[r(15)=.89, p<.001]$ and the separation of the eyes $[r(15)=.85, p<.001]$. The third MDS dimension correlated significantly with the thickness of the top lip $[r(15)=.82, p<.001]$ as well as component of head shape $[r(15)=$ $-.73, p<.01]$. Finally, the fourth MDS dimension also correlated significantly with thickness of the top lip $[r(15)=-.72, p<.01]$ and with head shape $[r(15)=.70, p<.01]$. The face coordinates thus obtained were used in the subsequent analyses. All distances reported for these faces are the Euclidean distances between the compared faces.

The faces were paired during the experiments with 16 of the most common male American names, as confirmed by the 1990 census. These names were truncated to their one-syllable equivalents: Jim, John, Rob, Bill, Dave, Rich, Charles, Joe, Tom, Chris, Dan, Paul, Mark, Mike, and George. Stimuli were presented subtending a visual angle of $3.7^{\circ}$ on a $1,280 \times 1,024$ computer screen against a white background. The names were presented under the faces in black capital letters.

Procedure. Participants first completed 64 trials of a samedifferent task (see Figure $3 \mathrm{~A}$ ). This introductory part of the experiment was implemented to help better familiarize participants with the stimulus set. On each trial, an orienting stimulus $(+)$ was presented for $1 \mathrm{sec}$ at the center of the computer screen. After a 200-msec blank screen, a face was shown for $700 \mathrm{msec}$, followed by another $3 \mathrm{sec}$ of blank screen and the presentation of a second face. Each of the intervals described above was jittered by a period between 0 and $75 \mathrm{msec}$ because we intended comparison with

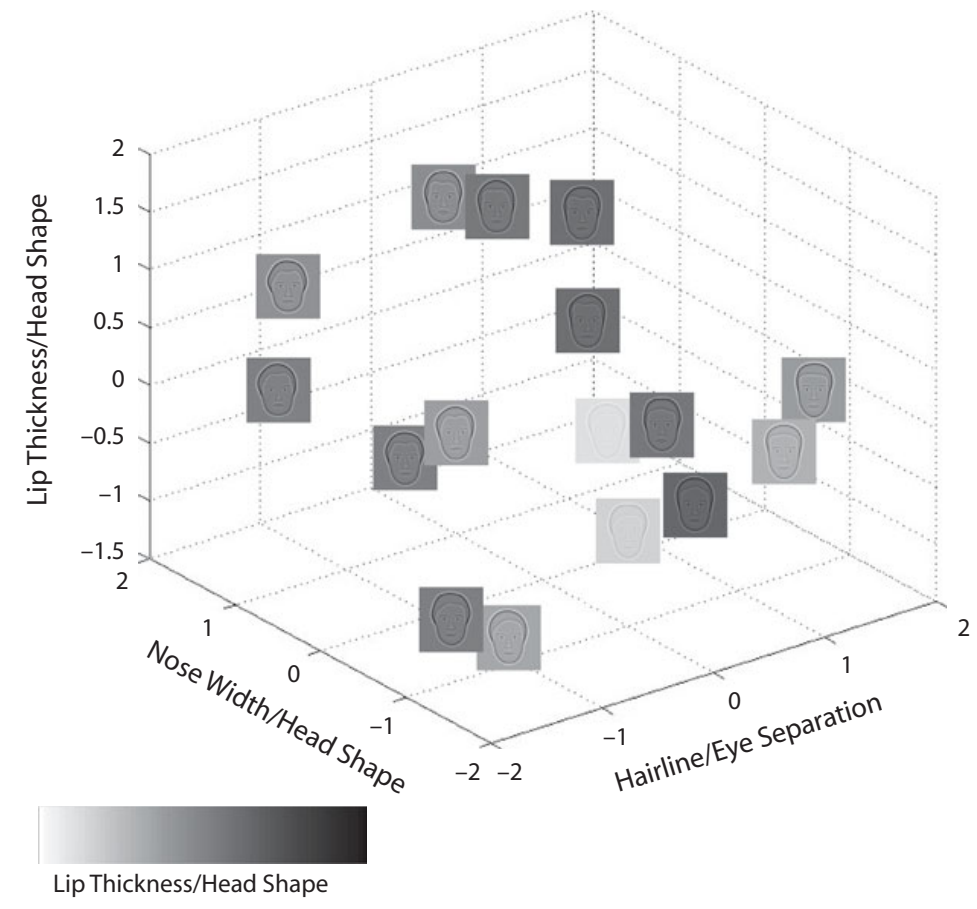

Figure 2. Face stimuli used in Experiment 1, plotted in their 4-dimensional MDSderived face space. The 4th dimension is indicated by the brightness of the face; negative coordinates in this dimension are associated with darker colors. 
A

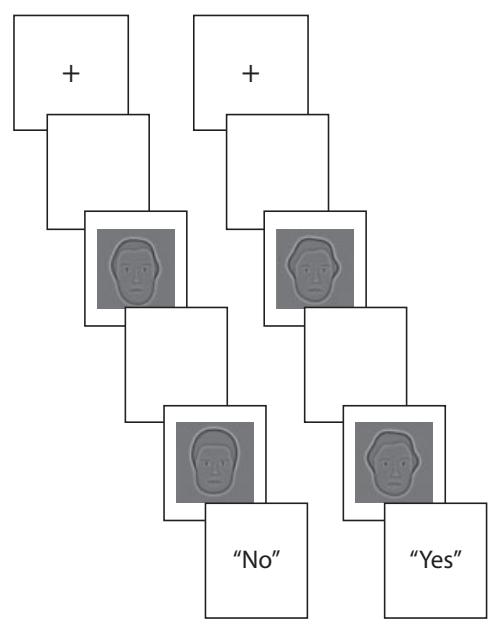

Same-Different Task
B

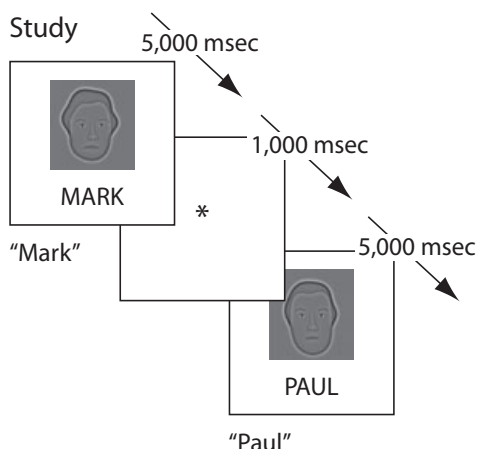

Study Phase
C

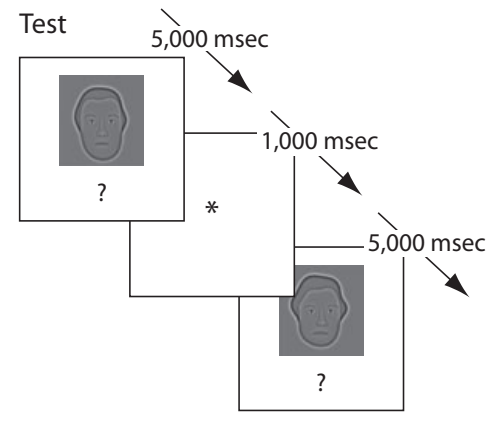

Figure 3. Illustration of the procedures used during the introductory same-different task (A), study phases (B), and test phases (C) of Experiment 1.

parallel EEG experiments for which the jitter is necessary to decorrelate brain signals across successive stimulus events. Participants reported whether the second face was the same or different from the first by pressing a designated button. The experimental procedure was delayed for as long as it took for the participant to respond, at which point the participant was informed whether the response was correct or incorrect and the next trial followed.

For every participant, the main experiment consisted of two study sets, each consisting of eight face-name associations. Facial stereotypes are known to be associated with certain male names (Lea, Thomas, Lamkin, \& Bell, 2007); therefore, we randomized the face-name pairings across participants in order to avoid spurious performance effects for particular face-name combinations. Each face-name pair was randomly assigned to one of the two study sets for every participant.

Each study set consisted of 10 blocks, each of which consisted of a study phase and a test phase. During the study phases, the eight face-name pairs were shown in random order for $5 \mathrm{sec}$ and their names were presented auditorily. During the test phases, faces were shown in random order for $5 \mathrm{sec}$ with a question mark underneath, and participants verbally recalled the corresponding names (Figures $3 \mathrm{~B}$ and $3 \mathrm{C}$ ). The testing computer digitally recorded participants' vocal responses. These recordings were analyzed offline to mark the onset time of each vocalization and to identify the participants' responses. The software used to record and analyze vocal responses (Geller, Schleifer, Sederberg, Jacobs, \& Kahana, 2007) has been used extensively in prior studies of spoken recall (Sederberg et al., 2006) and is freely available at pyepl.sourceforge.net.

\section{Results}

During the introductory 64-trial same-different task, hits and false alarms were respectively defined as trials on which participants correctly or incorrectly judged the two successively presented faces as being identical. Participants' hit rate $(\mathrm{HR})$ was $77 \%(S E M=5 \%)$ over the first 8 trials and $92 \%(S E M=3 \%)$ over the last 8 . Participants' false alarm rate (FAR) was $23 \%(S E M=4 \%)$ over the first 8 trials and $22 \%(S E M=5 \%)$ over the last 8 . Improvement in performance was significant for $\operatorname{HR}[t(24)=2.96, p<$ $.01]$ but not for FAR $[t(24)=0.13$, n.s.].

In the cued recall task, participants' performance improved from $28 \%$ correct recall on the first block to $83 \%$ correct recall on the 10 th block. Similarly, reaction time (RT) for correct recalls became gradually faster over the 10 test cycles. Descriptive data are given in Table 1 .

"Neighborhood" effect. Our primary question of interest was whether participants would exhibit worse associative recall performance for faces that had a greater number of similar "neighbors." Two faces were considered neighbors if they were previously judged to be highly similar to each other; that is, they were situated within a certain radius of each other in 4-D face space. The size of this radius was selected such that each face had one to seven neighbors within its respective study set (i.e., the set of faces also seen during the run), and the number of faces within this radius defined each face's "neighborhood density."

Table 1

Means and Standard Deviations of Recall Probability and Reaction Time, by Block in Cued Recall (Experiment 1)

\begin{tabular}{cccccc}
\hline & \multicolumn{2}{c}{$\begin{array}{c}\text { Recall } \\
\text { Probability }\end{array}$} & \multicolumn{2}{c}{$\begin{array}{c}\text { Reaction } \\
\text { Time (sec) }\end{array}$} \\
\cline { 2 - 3 } \cline { 5 - 6 } Block & $M$ & $S D$ & & $M$ & $S D$ \\
\hline 1 & .28 & .14 & 2.5 & 0.5 \\
2 & .44 & .15 & 2.5 & 0.7 \\
3 & .56 & .19 & 2.2 & 0.4 \\
4 & .66 & .17 & 2.2 & 0.4 \\
5 & .66 & .19 & 2.2 & 0.5 \\
6 & .70 & .16 & 2.1 & 0.4 \\
7 & .76 & .23 & 2.1 & 0.4 \\
8 & .81 & .17 & 2.1 & 0.4 \\
9 & .80 & .19 & 2.0 & 0.4 \\
10 & .83 & .20 & 2.1 & 0.5 \\
\hline
\end{tabular}



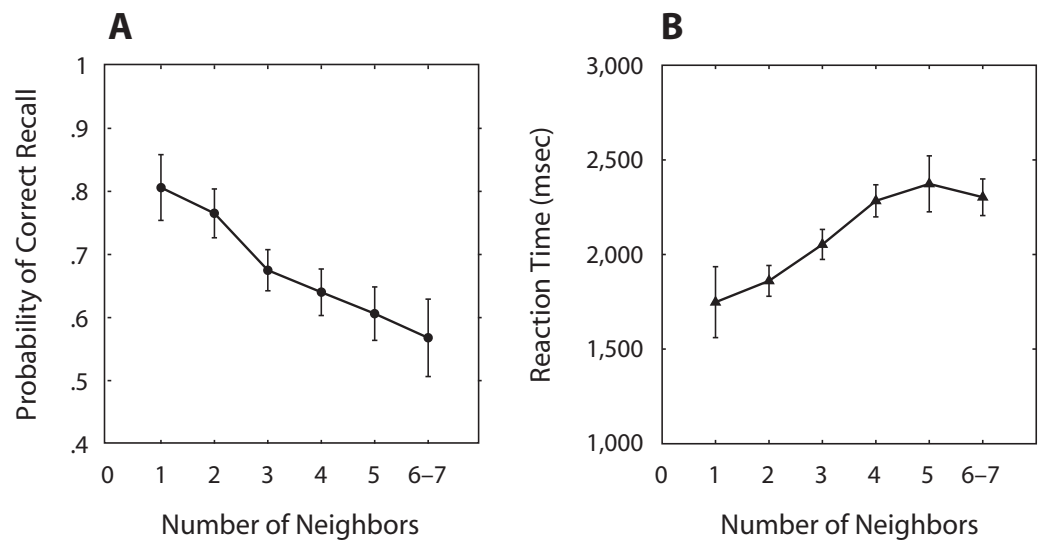

Figure 4. The "neighborhood" effect. Because of scarcity of data for faces with seven neighbors, faces with six and seven neighbors are collapsed into one bin. Error bars represent \pm 1 standard error of the mean.

At test, the more neighbors a cue face had within the study set the less likely it was that the correct name was recalled (see Figure 4). The 95\% confidence interval (CI) for the slope of the linear regression fitting probability of correct recall to neighborhood density was $(-0.09$, $-0.03)[t(24)=-4.07, p<.001]$. These recalls also were slower; the $95 \%$ confidence interval for the slope of the regression for RT on neighborhood density was $(83,249)$ $[t(24)=4.13, p<.001]$.

We performed one-way repeated measures ANOVAs across levels of neighborhood density ["low" (1-2 neighbors), "medium" (3-4 neighbors), "high" (5-7 neighbors)]. Two participants were excluded due to missing data. These ANOVAs confirmed the significance of the neighborhood effect. The difference across neighborhood density conditions for mean probability of correct recall was significant $[M($ low $)=.767, M($ medium $)=.664, M($ high $)=.595$; $\left.F(2,44)=12.99, M S_{\mathrm{e}}=.013, p<.001\right]$, and the same was true for mean RT $[M($ low $)=1,836 \mathrm{msec}, M($ medium $)=$ $2,158 \mathrm{msec}, M$ (high) $=2,365 \mathrm{msec} ; F(2,44)=10.55$, $\left.M S_{\mathrm{e}}=1.6 \times 10^{5} \mathrm{msec}, p<.001\right]$. Separate two-way ANOVAs (with three participants removed due to missing data) demonstrated that there was no significant interaction between number of neighbors (low, medium, high) and block ["early" (Blocks 1-5) vs. "late" (Blocks 6-10)], neither for probability of correct recall nor RT $[F(2,42)<1$, n.s., for both interactions].

We then explored whether this neighborhood effect was the result of associative interference from studying a particular face set, or a property of the global typicality or distinctiveness of a given face in the complete face space. Each face had one to seven neighbors within its own study set, but since each participant studied two different lists of eight face-name pairs during his session, each face could additionally be considered to have another set of neighbors in the set studied during the other half of his experimental session. We examined whether the neighborhood effect differed for faces with the same number of neighbors within their study set but a "low" (2-4) or "high" (5-7) number of neighbors in the set studied during the other half of the experimental session. Note that whereas participants were exposed to the whole face space at the start of the experiment (during the same-different task), one might still expect that this effect would be stronger during the second half of the experiment, after the participant had studied all of the faces and not just half of them.

We performed paired-sample $t$ tests (two-tailed) to compare the slopes of "neighborhood" regressions for "high" versus "low" other-half neighbor conditions. For probability of correct recall, no significant difference was found between the slopes of "neighborhood" regressions for high versus low other-half neighbors $[t(24)=0.42$, n.s.], and likewise for RT, no significant difference was found between the slopes of regressions for high versus low otherhalf neighbors $[t(24)=1.25$, n.s.]. When looking only at the second half of each participant's experimental session, once again no reliable differences were found between the slopes of regressions for high versus low other-half neighbor conditions, neither for probability of correct recall $[t(24)=0.83$, n.s. $]$ nor for RT $[t(24)=1.98$, n.s. $]$.

Two-way repeated measures ANOVAs revealed no significant effect of other-half neighbors (low, high) when crossed with within-half neighbors ["low"(1-3 neighbors), "high" (4-7 neighbors)], neither for probability of correct recall $[F(1,24)<1$, n.s.] nor for $\mathrm{RT}[F(1,24)<1$, n.s.]. The same was true if one looked only at the second half of each session (with 6 participants removed due to missing data), both for probability of correct recall $[F(1,18)<1$, n.s.] and for RT $[F(1,18)=1.48$, n.s.]. We concluded that the observed neighborhood density effects occurred independently of given faces' global typicality or distinctiveness. Rather, these effects were primarily the result of associative interference within a face's study set.

Additionally, we examined the possibility that cue faces with more neighbors came from lists that in general were more densely packed with faces (i.e., more crowded). In this case, the neighborhood effect could result not from these faces themselves being more difficult to learn rela- 
tive to other faces in their respective study lists, but instead from the relative difficulty of the study lists. To explore this issue, we classified each of the study lists into lower or higher density conditions. The classification was based on whether the dispersion among the face coordinates (the average of the distances between each of the eight list faces and the mean of the face coordinates for the given list) exceeded the median of the distribution across lists. If a study list did not vary significantly in face density, the hypothesis that the neighborhood effects we had observed were the result of this variation would be demonstrably false, since there is no variation. Below we show that even if there is a difference in density among lists, this does not affect the neighborhood density results.

We observed a neighborhood effect for both low- and high-density study lists, for the probability of correct recall-high, 95\% CI for the slope of the neighborhood density function $=(-.09,-.02)[t(16)=-3.69, p<$ $.01]$; low, $95 \%$ CI $=(-.10,-.03)[t(17)=-3.95, p<$ .01]. A similar effect was observed for RT-high, $95 \%$ $\mathrm{CI}=(69,217)[t(16)=4.10, p<.001]$; low, $95 \% \mathrm{CI}=$ $(85,353)[t(17)=3.47, p<.01]$. In addition, paired sample $t$ tests (two-tailed) were performed to compare the regressions for high- versus low-density lists, for the 10 (of 25) participants who by chance studied both a high-density list and a low-density list. For probability of correct recall, no significant difference was found between the slopes of neighborhood density regressions for high-density versus low-density lists $[t(9)=0.04$, n.s. $]$, and no significant difference was found between the intercepts for high density versus low density $[t(9)=0.14$, n.s.]. Likewise for RT, no significant difference was found between the slopes of regressions for high-density versus low-density lists $[t(9)=0.18$, n.s. $]$, and no significant difference was found between the intercepts for high density versus low density $[t(9)=0.38$, n.s. $]$. A possible caveat of this analysis is that the small number of participants limited statistical power; however, as explained below, data from Experiments 2 and 3 help to further rule out the alternative hypothesis considered here.

Intralist intrusions. Virtually all intrusions made by participants were ILIs - that is, incorrectly recalled names of other faces within the study set. During each test trial, participants could make an ILI by recalling any of the seven names of the other faces presented. For each ILI, we calculated the Euclidean distance in face space between the cue face and the face belonging to the incorrectly recalled name. Because the eight faces used in each run of the experimental session were selected randomly from a larger pool, ILIs to certain distances in similarity space were not always possible during a given session or study set. Therefore, the conditional probability of making an ILI was calculated by dividing the number of ILIs made by the number of possible ILIs for respective distance bins (see Figure 5). The 95\% CI for the slope of the linear regression fitting probability of ILI to Euclidean distance was $(-.03,-.02)[t(24)=-8.85, p<.001]$ and the $95 \%$ $\mathrm{CI}$ for the slope of the regression for the latencies of these intrusions was $(106,215)[t(24)=6.09, p<.001]$. These data demonstrate that intrusions are likely to come from nearby positions in similarity space.

Errors of memory versus perceptual confusion. The possibility remained that the similarity effects on memory we observed were the result of perceptual confusion between the highly similar faces. These two cognitive processes are inherently difficult to dissociate, but we attempted to do so by reexamining the main effects observed in Experiment 1 only for the subset of 8 participants that achieved $100 \%$ accuracy over the last eight trials of their introductory same-different task. Presumably, participants who achieved this accuracy criterion had little difficulty discriminating among the 16 synthetic faces.

With this reduced pool of participants, we still observed a significant neighborhood density effect. The 95\% CI for the slope of the regression of probability of correct recall
A

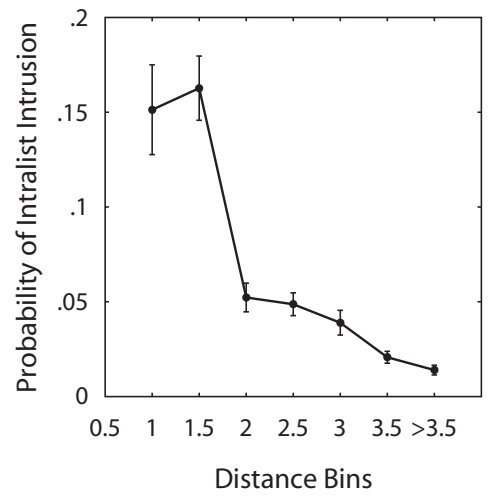

B

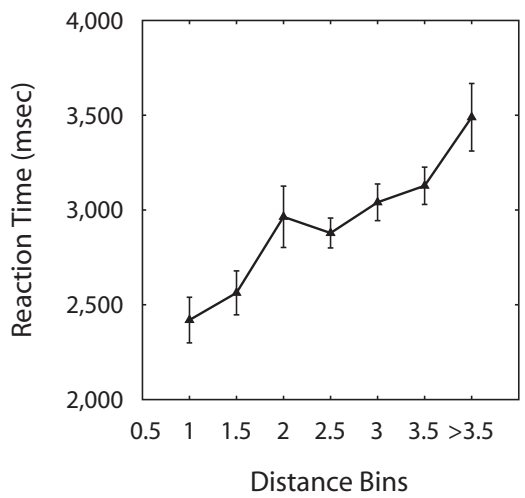

Figure 5. Intralist intrusions. (A) The conditional probability of making an intralist intrusion, as a function of the Euclidean distance between the face corresponding to the recalled name and the cue face. (B) The corresponding response latencies for these intrusions. Error bars represent \pm 1 standard error of the mean. 
on number of neighbors was $(-.11,-.02)[t(7)=-3.47$, $p<.05]$, and the $95 \% \mathrm{CI}$ for the slope of the regression for RT was $(42,223)[t(7)=3.45, p<.05]$. One-way repeated measures ANOVAs (with one participant excluded due to missing data) also showed a significant effect of neighborhood density (low, medium, or high) on both probability of correct recall $\left[F(2,12)=7.32, M S_{\mathrm{e}}=.013\right.$, $p<.01]$ and $\mathrm{RT}\left[F(2,12)=10.55, M S_{\mathrm{e}}=3.3 \times 10^{4}, p<\right.$ $.01]$. Additionally, we observed a significant similarity effect on ILIs. The $95 \%$ CI for the slope of the regression of probability of ILI on Euclidean distance was $(-.04,-.01)$ $[t(7)=-5.72, p<.001]$, and the $95 \% \mathrm{CI}$ for the slope of the regression of the latencies of these intrusions on distance was $(12,272)[t(7)=2.58, p<.05]$.

We then asked whether the neighborhood density effect could also be found for the subset of the eight worst discriminators. This set of participants, which averaged a 79\% HR and 38\% FAR over these last eight trials, presumably had the most difficult time discriminating between the faces, even after having been given significant exposure to them. If the neighborhood effect did indeed result in large part from problems of perceptual confusion rather than memory, then one would expect this effect to be greater for poor discriminators than for perfect discriminators. However, the slope of the linear regression fitting probability of correct recall to number of neighbors was virtually the same for poor discriminators $[M=$ $.063,95 \% \mathrm{CI}=(-.11,-.02)]$ and perfect discriminators $(M=.068)$, with no significant difference between the two groups $[t(14)=0.17$, n.s.]. Likewise for RT, the slope of neighborhood density regression was similar for poor discriminators $[M=211 \mathrm{msec}, 95 \% \mathrm{CI}=(-26,448)]$ versus perfect discriminators $(M=133 \mathrm{msec})$, and there was no significant difference between the groups $[t(14)=$ 0.73 , n.s.]. These data suggest that the similarity effects we observed reflected something beyond perceptual difficulty in discriminating between the faces.

\section{Discussion}

Experiment 1 demonstrated that the number of faces within a cue face's similarity neighborhood modulated performance in an approximately monotonic manner. Greater neighborhood density for a face was associated with lower accuracy and slower RT. This effect appears to have been the result of associative interference within the study set, independently of the global typicality of a given face within the context of the larger pool of faces. We also demonstrated that this effect was not a consequence of the overall crowdedness or density of lists being studied. To the extent that an ILI to Name B when cued with Face A is a measure of the interference of a Face B-Name B association with the correct recall of a Face A-Name A association, Experiment 1 also demonstrated that the closer that Face A and Face B were in similarity space, the more associative interference took place between the two pairs.

The faces in the set we used were highly similar (see Method), which raises the possibility that some memory effects we observed were actually the result of perceptual errors. Arguing against this alternative hypothesis, however, we restricted our analysis to the groups of
8 participants who best and worst learned to correctly discriminate between the faces during our introductory same-different task. With this approach, we found the same pattern of similarity-based associative interference. There are two additional reasons why perceptual confusions are an unlikely source of memory errors. First, nearest neighbors in face space were created to be one just-noticeable difference apart in face space at a presentation time of $110 \mathrm{msec}$ (see Method). A recent study by Habak, Wilkinson, and Wilson (2008) indicated that perceptual thresholds for the face stimuli we used decrease quite rapidly with additional viewing time in the time range between 110 and $1,000 \mathrm{msec}$. This suggests that it is likely that with the 700-msec viewing times we used in the present experiment, the faces are well discriminable. This is further corroborated by the fact that the average accuracy toward the end of the same-different task was $92 \%$, indicating that participants were generally able to discriminate between the faces fairly easily. We further addressed this issue in Experiment 2, which utilized a set of more distinctive faces.

\section{EXPERIMENT 2}

We next considered the possibility that the demonstrated effects of face similarity on associative recall were limited to the synthetic and highly confusable face set we employed in Experiment 1. To address this question, we conducted a methodologically similar experiment using a set of highly distinctive faces that more closely resembled actual photographs.

\section{Method}

Participants. Thirty-two undergraduates in a human memory lecture participated in the experiment as part of an optional in-class experiment.

Stimuli. For Experiment 2, a different set of 13 color renderings of Caucasian male faces from the Max Planck Institute (MPI) Tübingen database (O'Toole, Edelman, \& Bülthoff, 1998; for examples, see Figure 1B) were paired with 13 names out of the set of 16 utilized in Experiments 1 and 3. The faces used in this experiment were selected from among the 100 male faces in the MPI database (faces .kyb.tuebingen.mpg.de/). The renderings of these faces were derived from 3-D laser scans of the models' faces. Color information was also recorded; however, models were scanned without makeup and while wearing bathing caps (which were subsequently digitally removed). A normalization procedure was then used to bring each face into a common orientation and position. For the renderings used in this study, all faces were oriented toward the camera.

The similarity coordinates for these faces were determined using an MDS procedure. A separate sample of 69 participants drawn from a introductory psychology course rated the similarity of all possible pairs of faces on a scale from 1 (highly similar) to 10 (highly dissimilar). To remove intersubject variation in the $M$ and $S D$ of similarity ratings, we $z$-transformed each participant's ratings prior to forming the similarity matrix. Because this was a group experiment and the resultant data had many ties, individual differences MDS was not feasible. Therefore, the dissimilarity matrix consisting of the average ratings across participants was subjected to Sammon nonmetric MDS (Sammon, 1969) to yield a 2-D space for this face set. Sammon MDS differs from individual differences MDS in that it does not allow different perceptual weighting of the retrieved dimensions by individual participants. Instead, the solution is computed on the subject-averaged similarity matrix. Since 
there was no theory-based estimate of the number of dimensions of the face space, we determined the optimal number of dimensions by inspection of the Bayesian information criterion, or BIC (Lee, 2001), and found that five dimensions corresponded to the minimum BIC $(\mathrm{BIC}=742.53)$. We used the 5-dimensional MDS solution in the analyses presented below.

The names were presented under the faces in black capital letters, and the face-name stimuli were projected onto a screen in a lecture hall (visual angle varied between roughly $2^{\circ}$ and $9.5^{\circ}$, depending on where the participant was seated). In contrast to Experiments 1 and 3, no audio accompanied this experiment.

Procedure. In addition to utilizing a different set of faces, the procedure for Experiment 2 differed from that of Experiment 1 in five noteworthy ways. First, no same-different task preceded the experiment. Second, Experiment 2 utilized a single study set of 13 face-name pairs, instead of two study sets of 8. Third, Experiment 2 consisted of 5 study-test blocks, each employing the same set of 13 face-name pairs, instead of the 10 blocks per study set used in Experiment 1 . Fourth, participants provided written instead of spoken recall, and therefore we did not obtain RT data in this experiment. Finally, whereas for Experiment 1 names were randomly reassigned to different faces for each participant, for Experiment 2 each participant necessarily saw the same names paired with the same faces.

\section{Results and Discussion}

Although no RT data were recorded, in Experiment 2 we replicated the similarity effects that were observed in Experiment 1 for correct and incorrect recalls.

Learning curve. Participants correctly recalled 19\% of the faces' names on Block $1,42 \%$ on Block $2,56 \%$ on Block $3,72 \%$ on Block 4 , and $83 \%$ on Block 5 .

Neighborhood effect. The faces in this experiment each had between zero and five neighbors in the study set, with "neighbors" and "neighborhoods" being defined in the same manner as in Experiment 1. The slope of the linear regression fitting the probability of correct recall to the number of neighbors $(0-5)$ was calculated for each participant, and the $95 \% \mathrm{CI}$ for the slope of this regression was significantly negative $(-.067,-.036)[t(31)=$ $-6.85, p<.001]$. Then, separating the face-name pairs

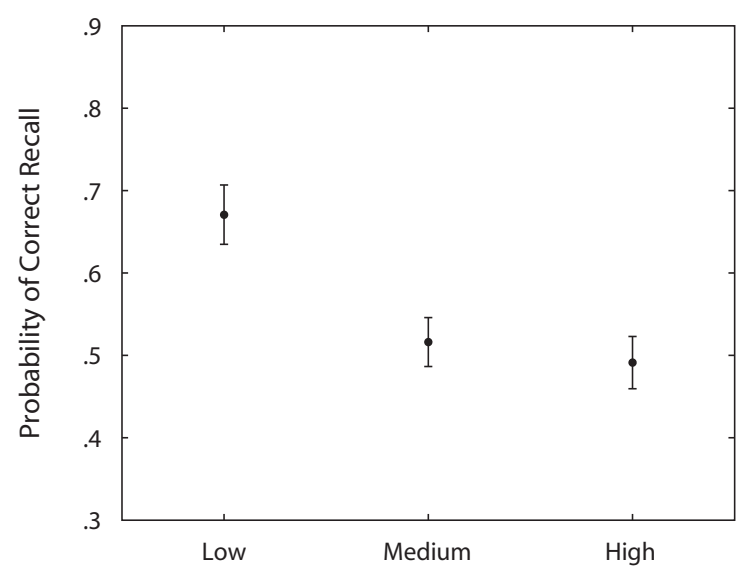

Number of Neighbors

Figure 6. The "neighborhood" effect in Experiment 2. Low $=$ 0-1 neighbors; medium $=2-3$; high $=4-5$. Error bars represent \pm 1 standard error of the mean.

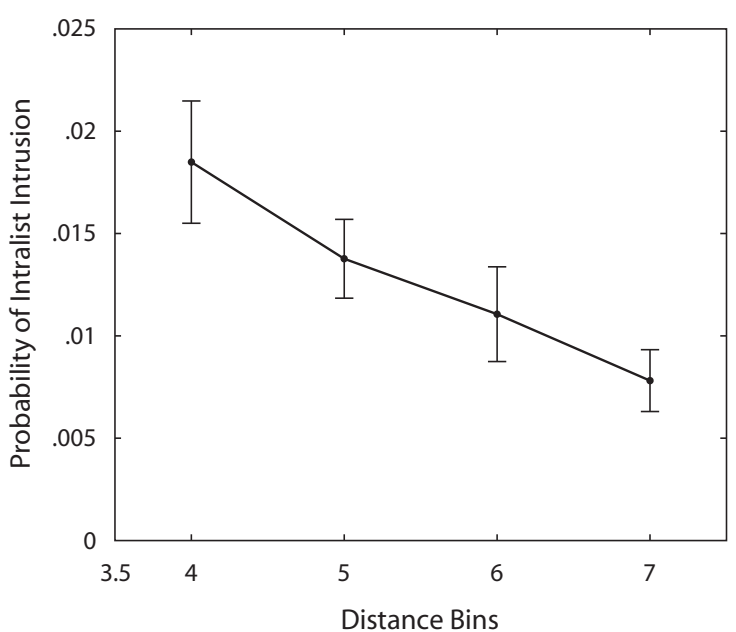

Figure 7. Conditional probability of intralist intrusion in Experiment 2 as a function of the Euclidean distance between the face corresponding to the recalled name and the cue face. Error bars represent \pm 1 standard error of the mean.

into "low," "medium," or "high" neighborhood density conditions ( $0-1,2-3$, or $4-5$ neighbors, respectively), we performed a repeated measures ANOVA for condition (low, medium, or high neighborhood density) on probability of correct recall, which also demonstrated a significant effect $\left[F(2,62)=26.29, M S_{\mathrm{e}}=.012, p<.001\right]$. The effects of neighborhood density on probability of correct recall are shown in Figure 6.

A separate two-way ANOVA revealed a significant interaction effect between neighborhood density condition and block ["early" (Blocks 1-3) vs. "late" (Blocks 4-5)] $[F(2,62)=6.84, p<.01]$. The slope of the linear regression fitting probability of correct recall to neighborhood density (low, medium, or high) was indeed more negative for early blocks $(M=-.11)$ versus later blocks $(M=$ $-.06)[t(31)=2.24, p<.05]$, although this neighborhood density effect retained statistical significance in the latter condition $[t(31)=-3.50, p<.01]$.

ILIs. The slope of the linear regression predicting conditional probability of ILI from the Euclidean distance between the cue face and the intrusion name's face was calculated for each participant. The $95 \%$ CI for the slope of this regression was significantly negative $(-.006,-.002)$ $[t(31)=-3.55, p<.01]$ (see Figure 7 ).

These results demonstrate that the similarity effects observed in Experiment 1 generalize to a more discriminable and arguably more ecologically valid set of faces. One might have argued that the similarity effects observed in Experiment 1 were actually the result of participants not being able to effectively discriminate between some faces in the set, within which all the faces were remarkably similar in appearance. This possibility was not entirely ruled out when we examined participants who performed with perfect discrimination toward the ends of their introductory same-different tasks. However, the faces employed in Experiment 2 were far more distinctive 
and readily distinguishable from one another than those used in Experiment 1. Although overall this made the task of learning their names much easier, the neighborhood density and similarity effects observed in Experiment 1 were replicated.

\section{EXPERIMENT 3}

Having established the effects of face similarity on recall accuracy and latency, we asked whether these effects could also be observed in an associative recognition task, using the well-controlled synthetic faces from Experiment 1 . To measure associative recognition at varying degrees of confidence, we asked the participants to make confidence judgments on a 6-point scale. At the end of the associative recognition task, we gave participants a final cued recall task to assess similarity effects following extensive exposure to the synthetic faces.

\section{Method}

Participants. Thirty-six undergraduate and graduate students of the University of Pennsylvania ( 10 male and 26 female) participated for payment and could additionally earn a bonus based on their accuracy and RTs. Each participated in one hour-long session.

Stimuli. The stimuli were identical to those in Experiment 1.

Procedure. Participants first engaged in the same 64-trial same-different task that was used in Experiment 1, to help better familiarize participants with the face set. The procedure for this task was identical, with the exception that these participants responded on the keyboard with a confidence judgment between 1 and 6 instead of a yes/no decision $(1=$ sure no; $6=$ sure yes $)$. This modification served to familiarize the participants with making confidence judgments, a procedure that they would use later in the experiment.

Experiment 3 utilized the synthetic faces from Experiment 1 but consisted of an associative recognition task at test, rather than cued recall. For each individual participant, each of the 16 faces in the pool was randomly paired with one of 16 names, and remained paired with the same name for the duration of the experiment. The experiment consisted of 10 study-test blocks. During each study phase, the procedure was identical to that utilized in Experiment 1, except for the increased number of pairs per block. For each test phase, half of the face-name pairs were correctly paired (targets) and half of the face-name pairs were rearranged (lures). The order of the study and test presentations and the correct or incorrect pairings were randomized across blocks and participants.

Table 2

Means and Standard Deviations of $\mathrm{Ag}$, Hit Rate, and False Alarm Rate, by Block for Associative Recognition (Experiment 3)

\begin{tabular}{|c|c|c|c|c|c|c|}
\hline \multirow[b]{2}{*}{ Block } & \multicolumn{2}{|c|}{$\mathrm{Ag}$} & \multicolumn{2}{|c|}{ Hit Rate } & \multicolumn{2}{|c|}{$\begin{array}{c}\text { False Alarm } \\
\text { Rate }\end{array}$} \\
\hline & $M$ & $S D$ & $M$ & $S D$ & $M$ & $S D$ \\
\hline 1 & .63 & .14 & .61 & .17 & .45 & .16 \\
\hline 2 & .71 & .15 & .70 & .19 & .40 & .18 \\
\hline 3 & .76 & .15 & .75 & .18 & .32 & .18 \\
\hline 4 & .82 & .12 & .81 & .19 & .29 & .16 \\
\hline 5 & .84 & .10 & .84 & .16 & .27 & .14 \\
\hline 6 & .85 & .10 & .84 & .11 & .27 & .15 \\
\hline 7 & .86 & .10 & .87 & .13 & .24 & .16 \\
\hline 8 & .88 & .10 & .88 & .12 & .20 & .14 \\
\hline 9 & .87 & .10 & .86 & .13 & .22 & .15 \\
\hline 10 & .87 & .14 & .88 & .13 & .22 & .19 \\
\hline
\end{tabular}

Table 3

Means and Standard Deviations of Reaction Times (in Seconds) As a Function of Block for the Four Response Types in Associative Recognition

\begin{tabular}{|c|c|c|c|c|c|c|c|c|}
\hline \multirow[b]{2}{*}{ Block } & \multicolumn{2}{|c|}{ Hits } & \multicolumn{2}{|c|}{ Misses } & \multicolumn{2}{|c|}{$\begin{array}{c}\text { Correct } \\
\text { Rejections }\end{array}$} & \multicolumn{2}{|c|}{$\begin{array}{c}\text { False } \\
\text { Alarms }\end{array}$} \\
\hline & $M$ & $S D$ & $M$ & $S D$ & $M$ & $S D$ & $M$ & $S D$ \\
\hline 1 & 2.4 & 0.5 & 2.8 & 0.6 & 2.6 & 0.6 & 2.6 & 0.8 \\
\hline 2 & 2.3 & 0.5 & 2.9 & 0.7 & 2.6 & 0.6 & 2.7 & 0.6 \\
\hline 3 & 2.3 & 0.5 & 2.9 & 0.8 & 2.6 & 0.5 & 2.8 & 0.9 \\
\hline 4 & 2.3 & 0.5 & 2.9 & 0.7 & 2.5 & 0.6 & 2.9 & 0.8 \\
\hline 5 & 2.3 & 0.4 & 2.8 & 0.8 & 2.5 & 0.6 & 2.7 & 0.7 \\
\hline 6 & 2.2 & 0.5 & 2.9 & 0.8 & 2.3 & 0.5 & 2.6 & 0.8 \\
\hline 7 & 2.2 & 0.5 & 3.0 & 0.9 & 2.3 & 0.5 & 2.5 & 0.7 \\
\hline 8 & 2.2 & 0.6 & 3.1 & 1.1 & 2.1 & 0.6 & 2.5 & 0.7 \\
\hline 9 & 2.1 & 0.5 & 2.8 & 1.2 & 2.3 & 0.6 & 2.5 & 0.8 \\
\hline 10 & 2.0 & 0.4 & 3.0 & 0.9 & 2.1 & 0.5 & 2.6 & 0.7 \\
\hline
\end{tabular}

The target-or-lure pair remained on the screen for $5 \mathrm{sec}$, and during this time participants responded on the keyboard with a confidence judgment on a scale from 1 to $6(1=$ sure incorrectly paired, $6=$ sure correctly paired). These confidence judgments would-in addition to providing for a sense of the confidence of the participant's responses - allow for the construction of receiver-operating characteristic (ROC) curves. If the participant failed to respond within $5 \mathrm{sec}$, the response was not recorded and the experiment moved on to the next trial. An asterisk was displayed for $1 \mathrm{sec}$ between trials.

At the conclusion of 10 study-test blocks, participants were presented with a brief cued recall task. The 16 faces were presented consecutively and in random order, with question marks underneath them. After $5 \mathrm{sec}$, each face disappeared from the screen, an asterisk was displayed for $1 \mathrm{sec}$, and the next face appeared on the screen. Participants attempted to recall and speak the name of each face into a microphone. Three of the participants did not participate in this segment of the experiment due to recording problems.

\section{Results}

During the introductory 64-trial same-different task, participants' HR was $82 \%(S E M=3 \%)$ over the first 8 trials and $93 \%(S E M=2 \%)$ over the last 8 . Participants' FAR was $27 \%$ ( $S E M=4 \%)$ over the first 8 trials and $16 \%$ $(S E M=4 \%)$ over the last 8 . This improvement in performance was significant for both HR $[t(35)=3.75, p<$ $.001]$ and FAR $[t(35)=2.43, p<.05]$.

For reasons discussed in greater detail below, we used $A g$ as an aggregate measure for performance in the associative recognition task ( $\mathrm{Ag}$ is determined by calculating the area underneath the ROC curve). $A g$ increased from .63 in the first block to .87 in the tenth and final block. Descriptive statistics for various metrics related to the learning curve are displayed in Tables 2 and 3.

As expected, on the basis of data from previous studies (e.g., Nobel \& Shiffrin, 2001), RTs varied significantly across the four response types $\left[F(3,105)=55.05, M S_{\mathrm{e}}=\right.$ $\left.4.9 \times 10^{4} \mathrm{msec}, p<.001\right]$. Post hoc comparisons (Bonferroni corrected, $p<.0083$ for all comparisons) revealed that RT for each of the response types was reliably different from RT for each of the other response types: Hits were fastest, followed by correct rejections, followed by false alarms, and misses had the slowest RTs.

In the following subsections we report tests of the effects of facial similarity on associative recognition performance. Additionally, we construct ROC curves, the 
Table 4

Statistics for the Linear Regressions of Dependent Variables on Neighborhood Density

\begin{tabular}{lclc}
\hline \multicolumn{1}{c}{ Predicted Variable } & Mean Slope & \multicolumn{1}{c}{$95 \% \mathrm{CI}$} & $t(35)$ \\
\hline Hit rate & -0.01 & $(-0.022,0.0002)$ & $-1.98^{\dagger}$ \\
False alarm rate & .00 & $(-0.010,0.016)$ & 0.50 \\
$\mathrm{Ag}$ & -0.02 & $(-0.036,-0.0002)$ & $-2.05^{*}$ \\
Avg. confidence, targets & -0.05 & $(-0.09,-0.01)$ & $-2.38^{*}$ \\
Avg. confidence, lures & 0.01 & $(-0.02,0.05)$ & 0.79 \\
RT(hits), msec & 51 & $(23,79)$ & $3.64^{* * *}$ \\
RT(misses), msec & 45 & $(-90,179)$ & 0.67 \\
RT(correct rejections), msec & -2 & $(-25,21)$ & -0.19 \\
RT(false alarms), msec & 4 & $(-43,50)$ & 0.16 \\
\hline$\dagger .05<p<.10 . \quad{ }^{*} p<.05$. & ${ }^{* *} p<.01 .{ }^{* * *} p<.001$. &
\end{tabular}

examination of which provides for a more thorough analysis of recognition performance as a function of response confidence.

Neighborhood effect. As in previous experiments, our question of primary interest was whether memory for a face-name association was affected by how many faces in the study set were in proximity to the face in similarity space. Each of the 16 faces had between 5 and 10 neighbors within the study set. We first separated the faces into "low" (5-6 neighbors), "medium" (7-8), and "high" (910) neighbor conditions.

To assess the reliability of the neighborhood effect, we regressed our aggregate performance measure, $\mathrm{Ag}$, on number of neighbors (low, medium, high). The neighborhood effect was significant for $\mathrm{Ag}$ (i.e., the fewer the neighbors within the study set, the higher the $\mathrm{Ag}$ at test) $[t(35)=-2.05, p<.05] .{ }^{1}$ However, when the effect was analyzed separately for HR and FAR, it was only marginally significant for $\mathrm{HR}[t(35)=-1.99, p=.055]$ and not significant for FAR $[t(35)=-0.50$, n.s.]. (See Table 4 for full descriptions of statistics for these regressions and those to follow.)

We also examined neighborhood density effects on confidence judgments and RT. Participants gave significantly higher confidence judgments to target pairs with lower neighborhood density (fewer neighbors) $[t(35)=$ $-2.38, p<.05]$. They also responded more quickly when correctly identifying these pairs as targets $[t(35)=3.64$, $p<.001]$. In the case of lure pairs, the effect of neighborhood density on confidence did not reach significance $[t(35)=0.79$, n.s.]. Similarly, RT for correct rejections was not significantly affected by the number of neighbors $[t(35)=-0.19$, n.s. $]$. There was no significant neighborhood density effect on RT for misses $[t(35)=0.67$, n.s. $]$ or false alarms $[t(35)=0.16$, n.s. $]$.

The effect of neighborhood density condition (low, medium, or high) on all of these metrics was also analyzed with repeated measures ANOVAs (see Table 5). Consistent with the previous set of analyses, one-way ANOVAs revealed a significant effect of neighborhood density on $A g$, RT for hits, and confidence for target trials. Neighborhood density effects on other metrics were not significant. Another set of two-way ANOVAs showed no significant interaction effects between neighborhood density (low, medium, high) and block number ["early"
(Blocks 1-5) vs. "late" (Blocks 6-10)] $[F(2,70)<1$ for all interactions, n.s.].

Confidence judgments. Because confidence judgments may provide a more sensitive metric of probe familiarity than HR or FAR, we examined the average confidence judgments participants gave, as a function of the Euclidean distance in similarity space between the probe face in the pair and the face belonging to the probe name (see Figure 8A). One would expect greater associative interference for a given face-name pair to result in a less confident - and for the purposes of this experiment, less accurate-response.

For this analysis, a target (correctly paired) pair was assigned a Euclidean distance of 0 , and lure (incorrectly paired) pairs could have varying distances. Lure trials were placed into four distance bins, and average confidence judgment was regressed on distance for each participant. Not surprisingly, the closer the lure face was to the correct face, the higher the judgment given. The $95 \%$ confidence interval for the slope of this line was $(-.54$, $-.33)[t(35)=-8.18, p<.001]$.

RT data were in line with the results observed in confidence judgments. For target trials, hits $(M=2,237 \mathrm{msec}$, $S D=421 \mathrm{msec})$ were significantly faster than misses $(M=2,858 \mathrm{msec}, S D=518 \mathrm{msec})[t(35)=11.99, p<$ $.001]$. For lure trials, RTs showed opposite trends, depending on whether they were false alarms (lure trials on which participants responded with confidence ratings between 4 and 6) or correct rejections (lure trials on which partici-

Table 5

Results of Repeated Measures ANOVAs Analyzing the Effects of Neighborhood Density (Low, Medium, or High) on Various Dependent Variables

\begin{tabular}{lcc}
\hline \multicolumn{1}{c}{ Predicted Variable } & $F(2,70)$ & $M S_{\mathrm{e}}$ \\
\hline Hit rate & 1.70 & 0.017 \\
False alarm rate & 0.39 & 0.008 \\
$A g$ & $3.42^{*}$ & 0.005 \\
Avg. confidence, targets & $5.67^{* *}$ & 0.123 \\
Avg. confidence, lures & 1.86 & 0.082 \\
RT(hits), msec & $10.51^{* * *}$ & $4.5 \times 10^{4}$ \\
RT(misses), msec & 0.75 & $1.5 \times 10^{5}$ \\
RT(correct rejections), msec & 0.10 & $3.0 \times 10^{4}$ \\
RT(false alarms), msec & 0.66 & $1.4 \times 10^{5}$ \\
\hline$\dagger .05<p<.10 .{ }^{*} p<.05 .{ }^{* *} p<.01$. & ${ }^{* * *} p<.001$. &
\end{tabular}



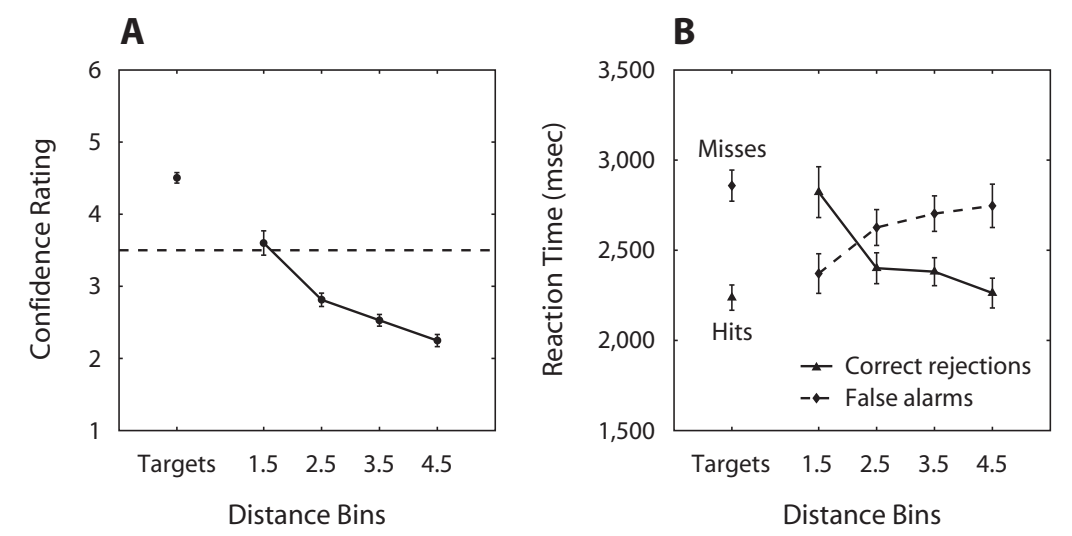

Figure 8. Average confidence judgment (A) and reaction time (B) as functions of Euclidean distance between the probe face and the face belonging to the probe name. In panel A, confidence judgments above the dotted line were defined as a response of "correct pairing," and judgments below the dotted line represented a response of "incorrect pairing." Error bars represent \pm 1 standard error of the mean.

pants responded with confidence ratings between 1 and 3 ). (See Figure 8B.) The shorter the distance, the faster the false alarm, and the $95 \%$ confidence interval for the slope of this regression was $(58,198)[t(35)=3.70, p<.001]$. Conversely, the shorter the distance, the slower the RT if the participant was to correctly reject the lure. The $95 \%$ confidence interval for the slope of this regression was $(-236,-80)[t(35)=-4.10, p<.001]$.

Final cued recall. We further examined to what extent the effects observed in direct cued recall (Experiments 1 and 2) could be extended to final cued recall in the present experiment. The ILI effects observed in Experiments 1 and 2 were replicated in the cued recall task presented at the end of each participant's experimental session. Figure 9A shows the probability of correct recall data along- side the data for conditional probability of ILI, on the same Euclidean distance scale. Corresponding RTs for these recalls are shown in Figure 9B.

Both of these similarity effects were significant. The $95 \% \mathrm{CI}$ for the regression of probability of ILI on distance was $(-.04,-.03)[t(32)=-8.42, p<.001]$. The $95 \%$ CI for the slope of the regression of intrusions' RT on distance was $(90,453)[t(32)=3.05, p<.01]$. We then recalculated these linear regressions when including data from correct recall trials along with the ILI trials. In this case, the $95 \%$ CI for the slope of the regression of probability of recall on distance was $(-.10,-.07)[t(32)=$ $-13.05, p<.001]$, and the $95 \%$ CI for the slope of the regression of RT on distance was $(253,388)[t(32)=9.71$, $p<.001]$.
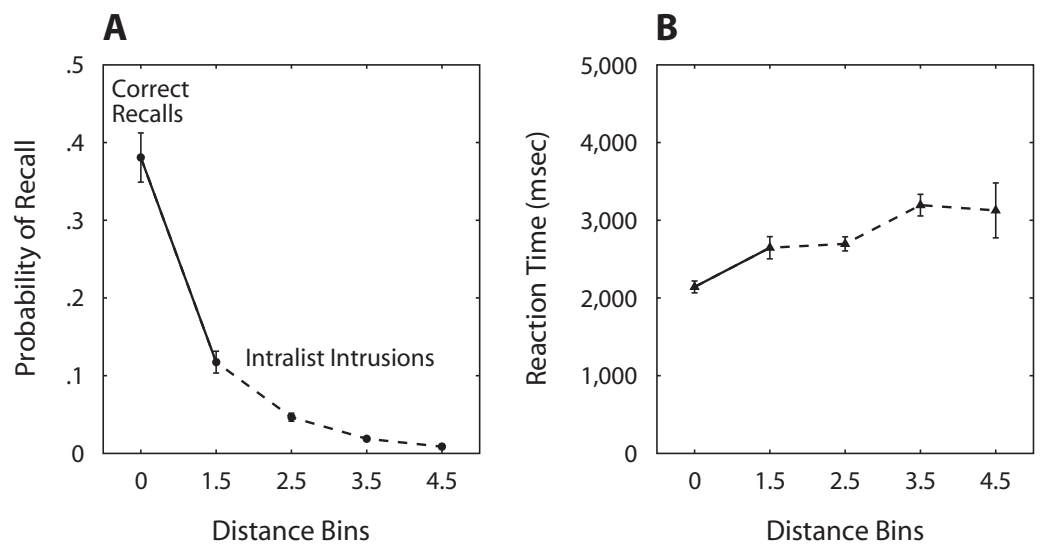

Figure 9. Final cued recall. (A) The conditional probability of recalling a name as a function of the Euclidean distance between the cue face and the corresponding face of the name (a correct recall has a distance of zero). (B) The corresponding reaction times of these recalls. Error bars represent \pm 1 standard error of the mean. 


\section{Discussion}

Experiment 3 demonstrated that the ability to recognize a face-name pair decreased as a function of the number of similar faces that were present on the study list, and how distant the probe face was in similarity space from the face that had been paired with the probe name at study. Although the observation of a neighborhood effect in recognition may seem intuitive given that we observed similar results in our cued recall experiments, the previous literature on verbal associations suggests that net performance in associative recognition may be immune to similarity-based interference. For instance, Dyne, Humphreys, Bain, and Pike (1990) presented participants with a verbal associative recognition task for which some pairs were studied among other pairs designed to produce interference (i.e., A-B, and later, $\mathrm{A}-\mathrm{C}$ in the same study list). Test items were constructed by rearranging some of the word pairs and leaving others intact. The experimenters found that both HR and FAR were greater for probe pairs that had been subject to interference at study. As a result, although cued recall performance had been reliably worse under associative interference conditions, they found no net effect in associative recognition, and this was put forth as evidence for a fundamental difference between associative recognition and associative recall (see also Postman, 1976). These results were consistent with the predictions of various summed similarity or global matching memory models (e.g., Gillund \& Shiffrin, 1984; Hintzman, 1984; Humphreys, Bain, \& Pike, 1989; Murdock, 1982). Another study, however, has found some evidence for similarity-based interference in associative recognition. Criss and Shiffrin (2004) had participants study lists of three categories of associations, varying the proportions of face-face, name-name, and face-name associations. The larger the proportion of facename associations was, the worse associative recognition performance for pairs from this category became. This was interpreted as an increase in similarity-based interference, because the larger the number of associations of the same category was, the stronger the interference was from competing pairs in this category. In contrast to our parametric analysis of similarity, the manipulation of similarity in this study was categorical.

The results observed in Experiment 3 demonstrated an effect on performance $(A g)$ resulting from associative interference. Dyne et al. (1990) noted that the concept of associative interference suggests that the learning of A-C somehow weakens the learning of A-B. In an associative recognition task, this would lead one to expect worse performance for target (intact) pairs in high-interference conditions and yet no marked effect on performance related to lure (rearranged) pairs. This is a scenario consistent with the results we observed when examining neighborhood density effects on HR, FAR, RT, and confidence in Experiment 3.

However, the interference effects we observed were of a subtly different variety than those searched for by Dyne et al. (1990). In our results, confidence and latency of responses for target and lure trials of varying distances suggested that the associative interference was attribut- able not only to a weakening of the association between Face A and Name A, but, relatedly, to partial association of Name A to the other competing faces studied in the list. With faces whose similarities were known, we observed in Experiments 1 and 3 that the relative magnitudes of these partial associations were a direct function of the Euclidean distances in similarity space between the target and competitor faces. For example, the confidence judgment data indicated that for lure pairs extremely close to what would have been the correct pairing, participants could typically not discriminate, since the average response was approximately halfway between a "yes" or "no" response.

\section{GENERAL DISCUSSION}

We observed that in both cued recall (Experiments 1 and 2) and associative recognition (Experiment 3 ), participants' memory for face-name pairs depended parametrically on the number of similar competitors within the study set. Specifically, faces that were distinctive among the study set yielded recall and recognition performance superior to that of faces that were situated in a dense region of the face similarity space. Furthermore, whenever participants made intrusions, they were more likely to come from nearby faces in face space. Thus, these findings show that similarity among faces for which names are being learned plays a major role in modulating the efficacy of an imperfect memory system during this task.

Although similarity effects have long been known to play an important role in a wide range of memory tasks (Kahana, in press) the dominance of verbal materials in associative memory studies has made it difficult to examine similarity effects parametrically. By employing a special class of difficult-to-verbalize visual stimulinamely, faces - and by using similarity ratings to obtain a multidimensional scaling solution for these stimuli, we were able to more precisely characterize the role of similarity in associative memory. We can thus go beyond the qualitative observation that highly similar stimuli produce greater interference than weakly similar stimuli do, by illustrating the functional relation between similarity and a variety of measures of memory performance, including recall accuracy and latency, intrusion rates, HRs, FARs, and response confidence.

One of our major research questions was whether interference effects would differ between associative recognition and cued recall, as had been proposed previously (Postman, 1976). Yet, the interference effects that we observed were quite similar across the two tasks. This is further evidence for cognitive similarity between these two memory tasks (Nobel \& Shiffrin, 2001).

Finally, our data suggest that these interference effects were most likely not an artifact of the difficult and abstract face set we used in Experiments 1 and 3, but are probably generalizable to the everyday task of learning the names of actual faces. The faces we employed in Experiments 1 and 3 were more difficult to tell apart than are most faces we encounter in everyday life. However, other studies support the claim that-especially when one removes 
distinguishing features such as hairstyle, facial hair, and skin pigmentation - the general ability to discriminate between faces viewed in isolation is cognitively complex and specifically vulnerable to a variety of perceptual manipulations (for an extensive discussion of various factors related to face processing and recognition, see Sinha, Balas, Ostrovsky, \& Russell, 2006). Nonetheless, even when we restricted our analysis to those participants who were able to discriminate among the faces without making any errors on eight consecutive trials, we observed the same neighborhood density effects (see Experiment 1). Furthermore, Experiment 2 demonstrated that even when discrimination between faces in a study set is made easier, the effects of similarity on associative memory are still quite apparent. This helps to support the claim that these memory effects are dissociable from perceptual confusion.

When we attempt to associate a name - an identitywith a person, we probably rely more than we realize on features other than the person's face. The person's hairstyle, the clothes he typically wears, his physical stature, semantic information known about the person, and the context within which we typically encounter the person all provide cues and mediating associations for the identification of a person and retrieval of his name (see Ganel \& Goshen-Gottstein, 2002, and Goshen-Gottstein \& Ganel, 2000 , for examples of how hair, for instance, is used heuristically to process faces). Using a combination of cues may be more reliable than relying on facial features alone. In Experiments 1 and 3, the presented face was accompanied by a spoken name, but the voice was identical for every face. We expect that if these experiments had been performed with distinctive voices for each face, participants would have learned the associations more easily. In essence, the distinctiveness of the additional auditory information would act to reduce to similarities among the name representations (and possibly even among the face representations). If one assumes that voice, clothing, and so on are additional elements in the similarity vector, which would be auto-associated upon learning, these additional features should facilitate performance on both recall and recognition tasks (cf. Polyn, Norman, \& Kahana, 2008; Underwood, 1969). This hypothesis could be tested by future experiments or simulations.

We interpret the data from all three experiments as indicating that the associative interference resulted from names being partially associated with faces in the study set other than the correct face. Because the extent of an incorrect partial association was a function of how near in similarity space the also-studied face was to the correct face, learning was weaker for faces with a greater number of competing faces nearby in similarity space. Within an experiment, all face-name pairs were studied among lists of the same size, but performance suffered most for those faces with greater within-study-set neighborhood density.

This interpretation comports well with attribute similarity and neural network models of associative memory (Bogacz, Brown, \& Giraud-Carrier, 2001; Hintzman, 1988; Humphreys, Pike, Bain, \& Tehan, 1989; Kahana, Rizzuto, \& Schneider, 2005; Murdock, 1982; Nosofsky, 1991; Rizzuto \& Kahana, 2001). According to these mod- els, items in memory are represented either as vectors of attribute values or as patterns of neural firing rates. Both cued recall and associative recognition rely on the formation and retrieval of associative structures binding the constituent vectors. It can easily be shown that similarity increases the interference between item representations during the associative retrieval process (e.g., Monaco, Abbott, \& Kahana, 2007; Murdock, 1989, 1992). Applying computational memory models to the similarity effects observed in both cued recall and associative recognition constitutes an important direction for future work.

\section{AUTHOR NOTE}

The authors acknowledge support from CELEST Grant SBE 0354378, Conte Center Grant P50 MH062196, NIH Grants RO1 MH061975, MH068404, EY002158, and MH055678, and the University of Pennsylvania Vagelos Fund. Correspondence concerning this article should be addressed to M. J. Kahana, Department of Psychology, University of Pennsylvania, 3401 Walnut St., Rm. 302C, Philadelphia, PA 19104 (e-mail: kahana@psych.upenn.edu).

\section{REFERENCES}

Bogacz, R., Brown, M. W., \& Giraud-Carrier, C. (2001). Model of familiarity discrimination in the perirhinal cortex. Journal of Computational Neuroscience, 10, 5-23.

Bower, G. H., Thompson-Schill, S., \& Tulving, E. (1994). Reducing retroactive interference: An interference analysis. Journal of Experimental Psychology: Learning, Memory, \& Cognition, 20, 51-66.

BusEy, T. A., \& TunNiclifF, J. L. (1999). Accounts of blending, distinctiveness, and typicality in the false recognition of faces. Journal of Experimental Psychology: Learning, Memory, \& Cognition, 25, 1210-1235.

Criss, A. H., \& Shiffrin, R. M. (2004). Pairs do not suffer interference from other types of pairs or single items in associative recognition. Memory \& Cognition, 32, 1284-1297.

Dyne, A. M., Humphreys, M. S., Bain, J. D., \& Pike, R. (1990). Associative interference effects in recognition and recall. Journal of Experimental Psychology: Learning, Memory, \& Cognition, 16, 813-824.

Feldman, S. M., \& Underwood, B. J. (1957). Stimulus recall following paired-associate learning. Journal of Experimental Psychology, 53, 11-15.

Fraas, M., Lockwood, J., Neils-Strunjas, J., Shidler, M., KrikoRIAN, R., \& WeIler, E. (2002). "What's his name?" A comparison of elderly participants' and undergraduate students' misnamings. Archives of Gerontology \& Geriatrics, 34, 155-165.

Ganel, T., \& Goshen-Gottstein, Y. (2002). Perceptual integrality of sex and identity of faces: Further evidence for the single-route hypothesis. Journal of Experimental Psychology: Human Perception \& Performance, 28, 854-867.

Geller, A. S., Schleifer, I. K., Sederberg, P. B., Jacobs, J., \& KAhAnA, M. J. (2007). PyEPL: A cross-platform experimentprogramming library. Behavior Research Methods, 39, 950-958.

GiBson, E. J. (1940). A systematic application of the concepts of generalization and differentiation to verbal learning. Psychological Review, 47, 196-229.

Gillund, G., \& Shiffrin, R. M. (1984). A retrieval model for both recognition and recall. Psychological Review, 91, 1-67.

Goshen-Gottstein, Y., \& Ganel, T. (2000). Repetition priming for familiar and unfamiliar faces in a sex-judgment task: Evidence for a common route for the processing of sex and identity. Journal of Experimental Psychology: Learning, Memory, \& Cognition, 26, 1198-1214.

Habak, C., Wilkinson, F., \& Wilson, H. R. (2008). Aging disrupts the neural transformations that link facial identity across views. Vision Research, 48, 9-15.

HintzMAn, D. L. (1984). MINERVA 2: A simulation model of human memory. Behavior Research Methods, Instruments, \& Computers, 16, 96-101.

HinTZMAN, D. L. (1988). Judgments of frequency and recognition memory in a multiple-trace memory model. Psychological Review, 95, 528-551. 
HoCKLEY, W. E. (2008). Memory search: A matter of time. In J. H. Byrne (Ed.), Learning and memory: A comprehensive reference (Vol. 2, pp. 417-444). Amsterdam: Elsevier.

Humphreys, M. S., Bain, J. D., \& Pike, R. (1989). Different ways to cue a coherent memory system: A theory for episodic, semantic, and procedural tasks. Psychological Review, 96, 208-233.

Humphreys, M. S., Pike, R., Bain, J. D., \& Tehan, G. (1989). Global matching: A comparison of the SAM, Minerva II, Matrix, and TODAM models. Journal of Mathematical Psychology, 33, 36-67.

Kahana, M. J. (in press). Foundations of human memory. Oxford: Oxford University Press.

Kahana, M. J., \& Bennett, P. J. (1994). Classification and perceived similarity of compound gratings that differ in relative spatial phase. Perception \& Psychophysics, 55, 642-656.

Kahana, M. J., Rizzuto, D. S., \& Schneider, A. (2005). Theoretical correlations and measured correlations: Relating recognition and recall in four distributed memory models. Journal of Experimental Psychology: Learning, Memory, \& Cognition, 31, 933-953.

Kahana, M. J., \& Sekuler, R. (2002). Recognizing spatial patterns: A noisy exemplar approach. Vision Research, 42, 2177-2192.

KePPEL, G. (1968). Retroactive and proactive inhibition. In T. R. Dixon \& D. L. Horton (Eds.), Verbal behavior and general behavior theory (pp. 172-213). Englewood Cliffs, NJ: Prentice Hall.

KNAPP, B. R., Nosofsky, R. M., \& Busey, T. A. (2006). Recognizing distinctive faces: A hybrid-similarity exemplar model account. Memory \& Cognition, 34, 877-889.

Lea, M. A., Thomas, R. D., Lamkin, N. A., \& Bell, A. (2007). Who do you look like? Evidence for facial stereotypes for male names. Psychonomic Bulletin \& Review, 14, 901-907.

LEE, M. D. (2001). Determining the dimensionality of multidimensional scaling representations for cognitive modeling. Journal of Mathematical Psychology, 45, 149-166.

Levitt, H., \& Goss, A. E. (1961). Stimulus attributes and drive in paired-associate learning. Journal of Experimental Psychology, 62, 243-252

Loffler, G., Yourganov, G., Wilkinson, F., \& Wilson, H. R. (2005). fMRI evidence for the neural representation of faces. Nature Neuroscience, $8,1386-1390$.

McGeoch, J. A. (1942). The psychology of human learning: An introduction. New York: Longmans, Green \& Co.

Monaco, J. D., Аввotт, L. F., \& Kahana, M. J. (2007). Lexicosemantic structure and the word-frequency effect in recognition memory. Learning \& Memory, 14, 204-213.

MurdocK, B. B. (1982). A theory for the storage and retrieval of item and associative information. Psychological Review, 89, 609-626.

Murdock, B. B. (1989). Learning in a distributed memory model. In C. Izawa (Ed.), Current issues in cognitive processes: The Tulane Flowerree Symposium on Cognition (pp. 69-106). Hillsdale, NJ: Erlbaum.

Murdock, B. B. (1992). Item and associative information in a distributed memory model. Journal of Mathematical Psychology, 36, 68-99.

Nelson, D. L., Bajo, M., McEvoy, C. L., \& Schreiber, T. A. (1989). Prior knowledge: The effects of natural category size on memory for implicitly encoded concepts. Journal of Experimental Psychology: Learning, Memory, \& Cognition, 15, 957-967.

Nelson, D. L., McKinney, V. M., Gee, N. R., \& Janczura, G. A. (1998). Interpreting the influence of implicitly activated memories on recall and recognition. Psychological Review, 105, 299-324.

Newman, S. E., \& Buckhout, R. (1962). S-R and R-S learning as functions of intralist similarity. American Journal of Psychology, 75, 429-436.

Nobel, P. A., \& Shiffrin, R. M. (2001). Retrieval processes in recognition and cued recall. Journal of Experimental Psychology: Learning, Memory, \& Cognition, 27, 384-413.
NOSOFSKY, R. M. (1991). Tests of an exemplar model for relating perceptual classification and recognition memory. Journal of Experimental Psychology: Human Perception \& Performance, 17, 3-27.

Osgood, C. E. (1949). The similarity paradox in human learning. Psychological Review, 56, 132-143.

O'Toole, A. J., Edelman, S., \& Bülthoff, H. H. (1998). Stimulusspecific effects in face recognition over changes in viewpoint. Vision Research, 38, 2351-2363.

Polyn, S. M., Norman, K. A., \& Kahana, M. J. (2008). Episodic and semantic organization during free recall: The control of memory search. Manuscript submitted for publication.

Postman, L. (1976). Interference theory revisited. In J. Brown (Ed.), Recall and recognition. London: Wiley.

Rizzuto, D. S., \& Kahana, M. J. (2001). An autoassociative neural network model of paired-associate learning. Neural Computation, 13, 2075-2092

RoBinson, E. S. (1927). The "similarity" factor in retroaction. American Journal of Psychology, 39, 297-312.

SAMMON, J. W. (1969). A nonlinear mapping for data structure analysis IEEE Transactions on Computers, C-18, 401-409.

Sederberg, P. B., Gauthier, L. V., Terushin, V., Miller, J. F., BarNATHAN, J. A., \& KahANA, M. J. (2006). Oscillatory correlates of the primacy effect in episodic memory. NeuroImage, 32, 1422-1431.

Sinha, P., Balas, B., Ostrovsky, Y., \& Russell, R. (2006). Face recognition by humans: Nineteen results all computer vision researchers should know about. Proceedings of the IEEE, 94, 1948-1962.

SkaGgs, E. B. (1925). Further studies in retroactive inhibition. Psychological Monographs, 34, 1-60.

TaKane, Y., Young, F. W., \& DE Leeuw, J. (1977). Nonmetric individual differences multidimensional scaling: An alternating least squares method with optimal scaling features. Psychometrika, 42, 7-67.

UNDERWOOD, B. J. (1953). Studies of distributed practice: VIII. Learning and retention of paired nonsense syllables as a function of intralist similarity. Journal of Experimental Psychology, 45, 133-142.

Underwood, B. J. (1969). Some correlates of item repetition in freerecall learning. Journal of Verbal Learning \& Verbal Behavior, 8 , 83-94.

van Vugt, M. K., Sekuler, R., Wilson, H. R., \& Kahana, M. J. (2008). Distinct electrophysiological correlates of proactive and similarity-based interference in visual working memory. Manuscript submitted for publication.

Verde, M. F., \& Rotello, C. M. (2004). Strong memories obscure weak memories in associative recognition. Psychonomic Bulletin \& Review, 11, 1062-1066.

Wilson, H. R., Loffler, G., \& Wilkinson, F. (2002). Synthetic faces, face cubes, and the geometry of face space. Vision Research, 42, 2909-2923.

Yonelinas, A. P. (1997). Recognition memory ROCs for item and associative information: The contribution of recollection and familiarity. Memory \& Cognition, 25, 747-763.

Yotsumoto, Y., Kahana, M. J., Wilson, H. R., \& Sekuler, R. (2007). Recognition memory for realistic synthetic faces. Memory \& Cognition, 35, 1233-1244.

\section{NOTE}

1. A ROC analysis confirmed that the effect of neighborhood density was consistent across varying decision criteria, and that the $z$-transformed ROC curve for associative recognition was approximately linear, and the slope had a $95 \%$ CI of $(1.10,1.28)$.

(Manuscript received June 14, 2007; revision accepted for publication April 23, 2008.) 